\title{
NASA D3R: 2.0, ENHANCED RADAR WITH NEW DATA AND CONTROL FEATURES
}

\author{
Mohit Kumar ${ }^{1}$,Shashank Joshil ${ }^{1}$, Manuel Vega ${ }^{1,2}$, V. Chandrasekar ${ }^{1}$, John W. Zebley ${ }^{2}$ \\ ${ }^{1}$ Colorado State University, Fort Collins, CO \\ ${ }^{2}$ NASA Goddard Space Flight Center, Greenbelt, MD
}

\begin{abstract}
The NASA dual-frequency, dual-polarization, Doppler radar (D3R) was developed to support development of algorithms and validation for the global precipitation measurement (GPM) missions dual-frequency precipitation radar (DPR). The D3R has participated extensively in various field campaigns in North America with geographic features covering both summer and winter climatic regimes. During the year 2017, D3R went through a major upgrade, specially with the digital receiver and waveform generation subsystems. In this work, the D3R systems upgrade will be discussed with a focus on key features of the new system. The new flexible architecture will enable new research capabilities that will be described.
\end{abstract}

Index Terms - D3R, radar, waveform generator, digital receiver, FPGA, waveforms.

\section{INTRODUCTION}

The NASA dual frequency, dual polarization doppler radar (D3R) was developed as a joint collaboration between NASA, Colorado state University and Remote Sensing Solutions. It allows for beam aligned, synchronized operation between $\mathrm{Ku}$ band $(13.91 \mathrm{GHz})$ and $\mathrm{Ka}$ band $(35.56 \mathrm{GHz})$ for the observation of a meteorological event. D3R forms an important ground validation tool for the GPM mission. The Ku band transmitter has peak power of 200 Watts [1] and Ka band has peak power of 40 Watts from solid-state transmitter. These power levels provide adequate sensitivity to measure light rain and snow. D3R also uses a multi-pulse scheme to mitigate the effects of blind range caused by longer pulses and subsequently using pulse compression to enhance the range resolution and sensitivity.

The D3R was deployed extensively to four field programs in the initial period ([2],[3]), and is due for an upgrade. One of the primary motivations for this upgrade is to enable additional radar sensitivity and improve range resolution to 30 meters [4]. In this paper, the D3R systems upgrade will be discussed with major focus on the system modifications.

Figure 1 features D3R in the recent deployment, after the upgrade, in an international collaborative experiment during

This work is sponsored by the NASA GPM project.

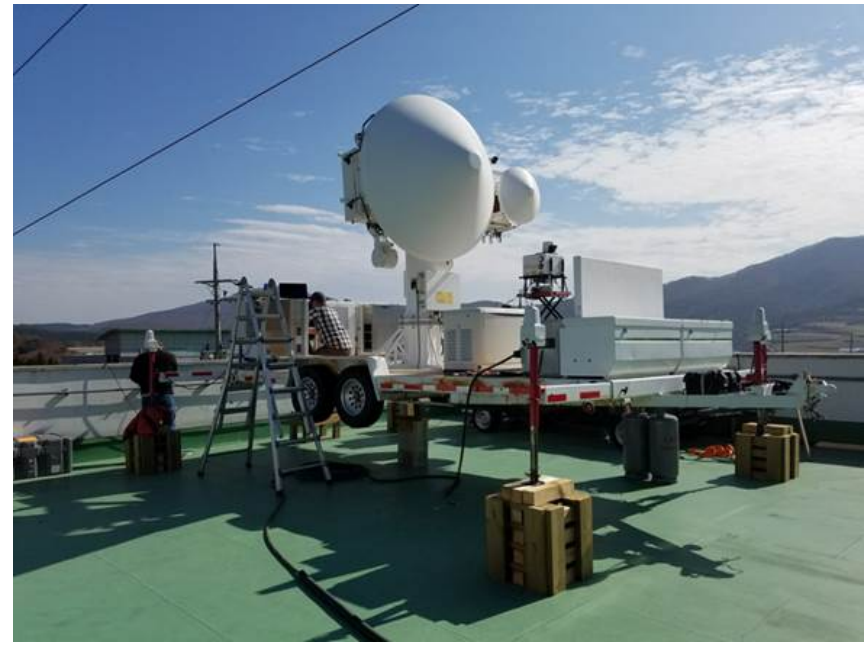

Fig. 1. The NASA D3R deployed at rooftop of DaeGwallyeong Regional Weather Office (DGW) in the daegwallyeong-myeon province of South Korea.

the PyeongChang 2018 Olympics and Paralympics winter games 2018 (ICEPOP) in South Korea.

As a part of the upgrade process, the Waveform Generator and digital receive sub-systems were changed along with their Intermediate Frequency (IF) electronics. As a result of change in IF sections, the clock generation scheme and the IF up-converter and down-converters have also been modified. Both the IF waveform generator and the digital receive sections have been packaged into a high speed digital module, based on virtex 6 Field Programmable Gate Arrays (FPGA). The ADCs and DACs have a high spur free dynamic range (SFDR), so as to enable sampling of intense storms to light cloud and snow observations. With high dynamic range, the sensitivity of the system can be improved, if the receive gain is tailored to toggle the lowest resolution bit of the ADC converter. This will enable the noise to be contained in the lowest bits (with a fine tuning of the receiver gain). Usually, if the SFDR is not good, additional spurious signals also be detected along with the weather echoes. These spurious signals may be due to the inter-modulation products of the mixer, which came in band due to aliasing and improper filtering or due to non-linearities of the ADC device itself. In this paper, 
we present analysis to contain these inter-modulation products and demonstrate the filtering requirements for such high dynamic range requirements. Also, we would be highlighting many such design features for the IF Stages, which has been incorporated in D3R as part of version 2.0 system for enhanced resolution, sensitivity and software control.

\section{DESIGN AND SIMULATIONS}

The digital module with DAC interface can generate upto a certain frequency range to maintain reasonable sampling rates with which digital in-phase and quadrature samples can be interfaced from FPGA to DAC device and also a feasible filter design. The IF stages and the analog bandwidth of these IF stages, were designed with the help of simulations of the mixer products and the rejections required in order to meet the dynamic range requirements. The challenge was to remove the Local Oscillator (LO) feedthrough, which is the high power spurious component, which can be trade-off with other intermodulation power levels. This made us to fix the analog bandwidth to get adequate rejection of LO feedthrough and other mixer products and harmonics.

With the simulations for the mixer products and the amount of gain required in the IF sections, the design evolved for the up-converter portion for the $\mathrm{Ku}$ and $\mathrm{Ka}$ band. The overall design philosophy was to segregate the LO generation portions to clock box and IF up-convert stages to Waveform box. The LO power requirements were satisfied partially at the clock box level and partially at the waveform generator level. Thus the design meets requirements of power to drive the transceiver inputs and maintains good spectral purity of signals (managed through adequate filtering stages). Based on simulations of mixer products and LO leakage, the filtering requirements were determined. These requirements are more stringent for the up-convert stages than the receive, as the power at each stage is high and most of the components in the chain operate near to $3 \mathrm{~dB}$ compression level.

After the gain and the mixing stages, we finally arrive at last IF stage on the Horizontal and Vertically Polarized receiver channels, further digitized by the ADC digital module. The receive path uses amplifier with flat gain (over our region of interest) and low noise. The high inter-modulation products of the mixer are suppressed by filters in the receive chain and also finally the spectrum is cleaned up by an anti-aliasing filter before ADC sampling. It should be made sure that the spurious components should not leak out even after coherent integration. The components of the receive chain have high individual Third Order Intercept points (OIP3) such that the resultant OIP3 is high. This has aided us in achieving high SFDR for the receiver. The products with high OIP3 consume high power and require cooling, thus there is a tradeoff between power requirements and OIP3.

Figure 2 shows the overall division of design and functionality in different sub-systems. These sub-systems were

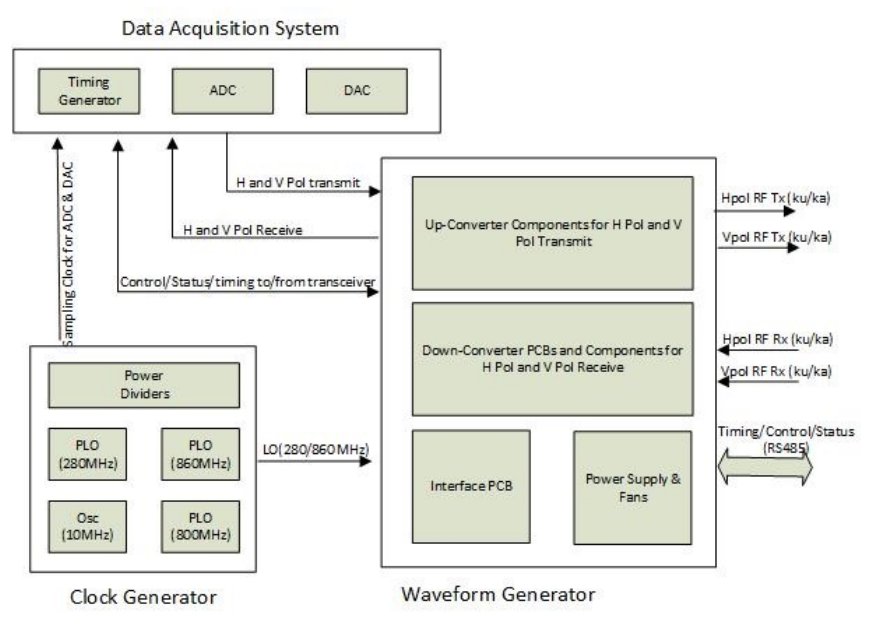

Fig. 2. Design of IF stages for ku and ka band, showing the different sub-systems involved.

designed for $\mathrm{ku}$ and $\mathrm{ka}$ band with different frequencies at IF.

The IF frequency planning is accomplished after simulations and availability of synthesizer modules for those frequencies. Appropriate amplification or attenuation stages are added to meet the requirement of drive levels for the up and down converter stages. A basic block diagram expaining this, is in Figure 3. All of these components are part of the clock box.

\subsection{Board Layout and Shielding Enclosures}

The board layout is done keeping in mind the RF design and layout guidelines and appropriate shielding enclosures are used to curb EMI to/from these boards. We also designed a signal interface board to drive RS485 signals and receive them and convert to LVDS format, for the digitizer module. Transient suppressors were added to the 485 lines, which traverse long distances. 1pps signal and sync-in are the coaxial inputs to this board and sync out is the coaxial output, from this board. The sync in- sync out interface is provided so as to maintain synchronization between ku and ka systems.

\subsection{Receive Gain}

The receiver gain is calculated based on the highest strength signal strength expected from nearby strong scatterers which can be resolved, without saturating the ADC. In our case, for the calibration exercise, we mount a corner reflector, around $500 \mathrm{~m}$ away from the radar. This corner reflector is on top of a tall platform, to clear the sidelobe returns from ground, and for achieving a high enough SNR, for reflectivity calibration. This high received power from this corner reflector has to be adequately resolved, if we need to do the calibration exercise for receive and transmit calibration. For achieving this, not only ADC full scale should be sufficient to represent 


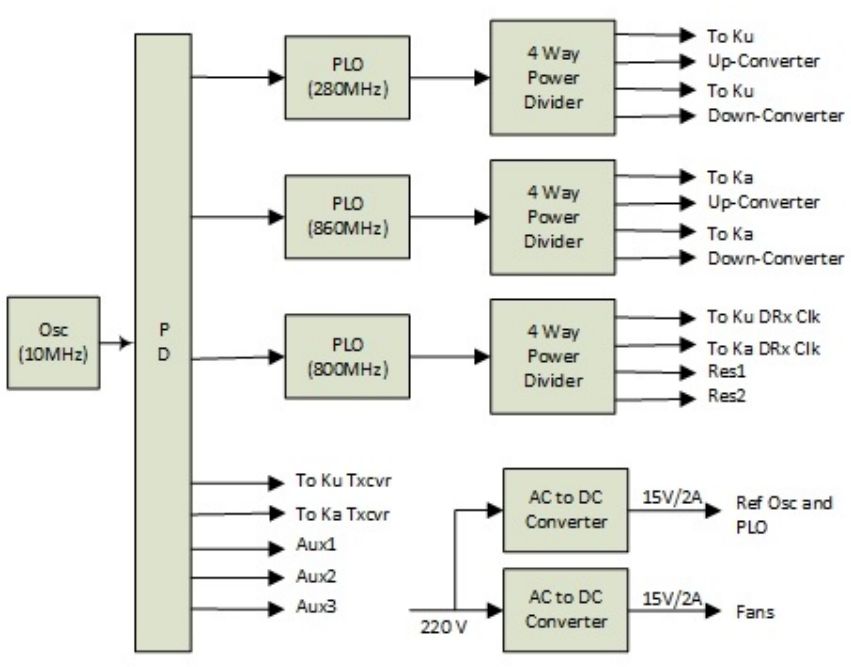

Fig. 3. The basic block diagram showing synthesis of different IF frequencies and clocks for ku and ka sections.

this power and not saturate but also, the intermediate amplifiers should be operating in a linear region as well. With the received power as reference, to ensure that none of the intermediate amplifiers were saturated, we had set up attenuators in between and thus, the appropriate gain for the IF stages was set. After the gain of the receivers were set up, we could also calculate the noise power seen by the ADC. The receive gain in apportioned into various stages, so that the power and cooling requirements of the amplifiers at different stages, can be managed.

\section{ASSEMBLY, INTEGRATION AND TEST}

The waveform generator unit working at IF frequency, consists of $\mathrm{H} \mathrm{Pol}$ and V Pol receive sub-systems housed in two shielded enclosures per channel. It also has a timing and interface buffer board to transceivers Apart from this, this unit has the IF up-converters for driving the final RF stages in the transceiver unit. The clock unit comprises of clock synthesizers (PLL based) to multiply the basic reference clock to the IF frequencies of ku and ka systems. It also has the necessary power splitters to connect to IF receivers and up-converters. The basic oscillator is mounted on shock mounts to improve phase noise performance under vibration conditions. To regulate the overall temperature in the IF box, it has thermoelectric fans and temperature monitoring system to provide heating or cooling, for these sub-systems. Initially, the newly built systems were tested on bench standalone, and later these were integrated in the IF box of D3R. Recently, the newly built systems and D3R radar, after upgrade, was deployed in an international collaborative experiment (ICEPOP) for the 2018 winter olympics and paralympics, held in Pheongchang (South Korea).

\section{PRELIMINARY RESULTS}

The basic goal behind the upgrade, was to incorporate more flexibility into the digital hardware, making the digital subsystems like waveform generation, data acquisition and filtering, more tightly coupled and integrated into a single FPGA device. This would enable agility of waveform, filter coefficients, IF frequency, etc pulse-to-pulse, and also use of coding techniques. These techniques are known to improve upon second trip suppression (or second trip retrieval) and cross power isolation. Some of these techniques are in the process of testing in D3R radar. Another motivation behind the upgrade was to enhance the resolution of the radar to $30 \mathrm{~m}$ from the current resolution of $150 \mathrm{~m}$. With this high resolution mode, we would be able to observe the fine micro-physical properties of the storms and analyze them in a greater detail.

A highly flexible system such as D3R upgrade version 2.0, will allow trade-off between sensitivity and temporal resolution. A highly sensitive system looses out on temporal resolution (by synthesizing narrow band filters) and vice versa. The upgrade version will have high sensitivity mode (low range resolution) and a high temporal resolution mode, to fulfill both these requirements.

During the ICEPOP campaign conducted in Feb-March 2018, the upgraded D3R was deployed at Pheongchang region of South Korea, to improve our understanding of interaction between ocean and mountain region, on the formation of snow. With the dual-frequency operation and by using DFR (dual-frequency ratio) measurements, along with other dual-polarization moments, data from this campaign would be highly beneficial for these micro-physical aspects of snow formation and snow types, particularly, classification of various snow aggregate types. The observations for one of snow case in Pheongchang, is shown in figure 4.

\section{CONCLUSION}

The D3R upgrade with emphasis on system improvements, is discussed. The various features of upgrade are described, with reference to dynamic range and spectral purity, hence leading us towards filtering requirements and gain of the system. The design techniques used to achieve this, is discussed and finally assembly and integration in the radar, is also discussed. The new upgraded system was deployed for monitoring of snow storms for the winter olympic games.

\section{ACKNOWLEDGEMENTS}

The authors would like to thank David Wolff and Walter Petersen for their constructive suggestions throughout the course of the project. Also special thanks to Jim George, for his constant guidance and support throughout the upgrade. 

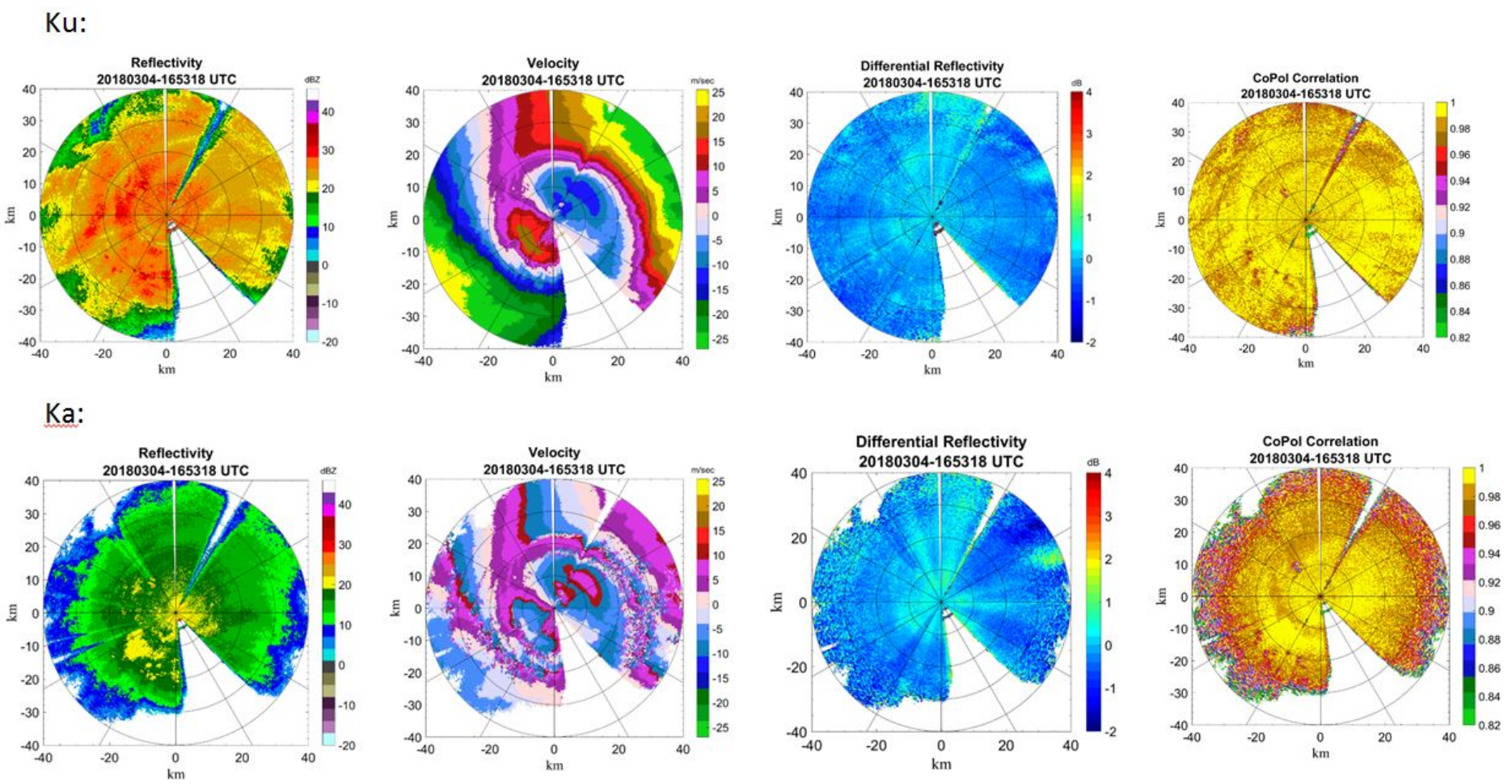

Fig. 4. The ku and ka band ppi observations of dual-pol variables for 4th March 2018 snow storm case around 16:53UTC.

\section{REFERENCES}

[1] Manuel A. Vega, V. Chandrasekar, James Carswell, Robert M. Beauchamp, Mathew R. Schwaller, and Cuong M. Nguyen, "Salient Features of the Dualfrequency, Dual-polarized, Doppler Radar for Remote Sensing of Precipitation," Radio Science, 2014.

[2] Robert M. Beauchamp, V. Chandrasekar, Haonan Chen, and Manuel Vega, "Overview of the D3R Observations during the IFloodS Field Experiment with Emphasis on Rainfall Mapping and Microphysics," Journal of Hydrometeorology, vol. 16, no. 5, pp. 2118-2132, 2015.

[3] V. Chandrasekar, Robert M. Beauchamp, Haonan Chen, Manuel Vega, Mathew Schwaller, Walter Petersen, and David Wolff, "DEPLOYMENT AND PERFORMANCE OF NASA D3R DURING GPM IPHEX FIELD CAMPAIGN," in Geoscience and Remote Sensing Symposium (IGARSS), 2015 IEEE International, 2015, pp. 26222625.

[4] M. Kumar, S. S. Joshil, V. Chandrasekar, R. M. Beauchamp, M. Vega, and J. W. Zebley, "Performance trade-offs and upgrade of nasa d3r weather radar," in 2017 IEEE International Geoscience and Remote Sensing Symposium (IGARSS), July 2017, pp. 5260-5263. 